\title{
Asphalt durability and self-healing modelling with discrete particles approach
}

\author{
V. Magnanimo, H. L. ter Huerne and S. Luding
}

Tire-Road Consortium, CTW, University of Twente, Netherlands - corresponding author: v.magnanimo@utwente.nl

\begin{abstract}
Asphalt is an important road paving material, where besides an acceptable price, durability, surface conditions (like roughening and evenness), age-, weather- and traffic-induced failures and degradation are relevant aspects. In the professional road engineering branch empirical models are used to describe the mechanical behaviour of the material and to address large-scale problems for road distress phenomena like rutting, ravelling, cracking and roughness. The meso-scopic granular nature of asphalt and the chemistry and mechanics of the bitumen layer between the particles is only partly involved in this approach. The discrete particle method is a modern tool that (first of all) allows for arbitrary (self-)organization of the asphalt meso-structure and for rearrangements due to compaction/cyclic loading. This is of utmost importance for asphalt during the construction phase and the usage period, in forecasting the relevant distress phenomena and understand their origin on the grain-, contact-, or molecular scales. Contact-forces that involve visco-elasticity, plasticity, friction and roughness are state-of-the art in fields like particle technology and can now be modified for asphalt and validated experimentally on small samples. The ultimate goal is then to derive micro- and meso-based constitutive models that can be applied to modelling behaviour of asphalt pavements on the larger scales. Using the new contact models, damage and crack formation in asphalt and their propagation can be modeled. Furthermore, the possibility to trigger selfhealing processes in the material is carefully investigated from a micro-mechanical point of view.
\end{abstract}

\section{Introduction}

Asphalt mixtures are composite materials that consist of solid particles, viscous binder/fluid (bitumen) and pores filled with air. When considering asphalt mixtures we should distinguish different states the mixture can be in: a) hot and noncompacted (construction phase, relatively loose particle matrix) and b) compacted and ambient temperature. During compaction of the mixture the relative contents of the different phases change: the initially loosely compacted material, the particle matrix changes (proximity of the particle skeleton) and air filled voids are squeezed out. The material compacts. The fluid in the mixture (that can be hot or cold, i.e. less or more viscous) lubricates the contact surfaces between the particles and can even make movement of the particles easier [1]. The multiphase material has different properties that depend on those of the original components, i.e. aggregate and mortar. The physical properties (e.g. shape, surface texture, size distribution, skeletal structure, moduli), but also the properties of the binder (e.g. grade, relaxation characteristics, cohesion) and binder-aggregate interactions (e.g. adhesion, absorption, physiochemical interactions) characterize the material behavior of the asphalt mixture. In addition, aggregate particles in the mixture have different shapes, surface texture and friction, and orientations, which make the description of the contacts between particles a challenge [2].

When looking at asphalt, it makes sense to distinguish between three different length scales, i.e. micro, meso- and macro-scale. The interaction between the mortar (composition of bitumen and the smallest particles) and the larger stones is defined as the micro-scale. The interaction of multiple stones of various sizes and the mortar is defined as the meso-scale. On the macro-scale, the behavior of the whole road is accounted for. Kinematics at different scales apparently governs the behavior of the material: to gain thorough knowledge of asphalt pavement behavior, one has to focus on all three length scales. Common practice is that in the professional asphalt branch, fundamental constitutive models that are able to describe the micro- and meso-mechanical behavior of the material are hardly used. Large-scale problems are addressed by using empirical models [1] for road distress phenomena like rutting, raveling, cracking and roughness. The meso-scopic granular nature of asphalt and the chemistry and mechanics of the (modified) bitumen 
layer between the particles is, because of the limitations of those empirical models, just partly involved within those techniques. Our ambitious goal is to bridge the gap between discrete and continuous, macro-concepts. The material behavior at the grain-scale can be combined with the granular structure in order to identify the contact law for the asphalt components and relate such kinematics with the macroscopic response at the larger scale of the road. Finding a micro-based model with predictive quality on the macro-level is the ultimate challenge.

On the particle-scale, the interaction of the bitumen with the grains and between the grains can be efficiently investigated using a Discrete Element Method (DEM) [3]. Discrete element methods simulate particulate systems by modeling the translational and rotational degrees of freedom of each particle using Newton's laws, and the forces are calculated associating proper contact models with each particle contact.

In the last twenty years, attempts for a micromechanical modeling of asphalt have been done by some researchers: a contact law for the behavior of two particles connected by a binder and elastic behavior of an assembly of bonded particles was proposed in Ref. [4]; a micromechanical description of rutting with intergranular and aggregate-binder interactions was given in Ref. [5]; as well as 2D modeling based on image processing [2]. DEM studies on cemented particulate materials include the work by Rothenburg et al. [5], Chang and Meegoda [6], Sadd and Dai [7], Buttlar and You [8] and Ullidtz [9]. A different numerical approach is used in [10], where the material is idealised with single sized perfect spheres bounded by mortar, and modeled by Finite Element Method. Nevertheless, a well established multiscale description for the constitutive behavior of particle-bitumen systems is still missing. Particularly, very limited work has been done on 3D modeling of the mixture, with proper visco-elasto-plastic, temperature-dependent contact/interaction models. Moreover, to our knowledge, no systematic numerical study on the microscopic processes that govern cracks (and eventually self-healing) in asphalt has been done. On the technological/methodological side, a general computational framework suitable for simulating the loading/cyclic and fracture/healing behavior of granular-bitumen composites is the need of the hour. The detection and analysis of damage as well as microscopic self-healing mechanisms have been achieved by particle based simulations already [11][12] so that these results now can be applied to asphalt and translated to a practicable continuum model with predictive quality, which will allow proposing and designing optimized materials with superior self-healing capabilities in the future.[13].

In this preliminary study an approach to modeling of cohesive, sintering and self-healing particulate materials is described [14]. The mechanical evolution in time of all the particles in the material is simulated in DEM using a modern version of the sintering contact model [11]. This contact model mimics the physical behavior of the interaction of the particles accounting for dissipative, elasto-plastic, adhesive, and frictional effects and their pressure dependence. It is by construction non-linear in its response to external load [11]. An additional temperature-dependence, as possibly resulting from diffusion of atoms and the resulting sintering effects, can be added on top of the elasto-plastic contact model [12].

Initially, some particle-samples are prepared by applying isotropic (hydrostatic) pressure. The spherical particles deform plastically at contact and stick to each other forming a solid sample with (after releasing pressure) zero confining stress - on which uni-axial tension or compression is applied. The numerical results are compared (qualitatively) with laboratory uni-axial tests from [15] on samples of dense asphalt concrete. The comparison is encouraging as the model is able to reproduce the typical softening behavior of asphalt under compression.

Large deformations of sintered samples will then lead to damage. In the final part of the work, self-healing of sintered particulate materials is studied using the DEM simulations. Loose particles can sinter due to their time-, stress- or temperature-dependent contact-properties and -forces. Self-healing of damaged samples can be activated through resintering, a process that globally increases further the contact adhesion between particles. The simplest self healing of particle contacts only involves the increase in contact strength due to re-sintering, without accounting in detail for all microscopic mechanisms involved. Experimental and numerical studies indicate that the net increase in strength depends on the sintering temperature and duration, see for example [12],[16],[17]. In the simulations, during the initial pressure sintering preparation, the optimal strength is not reached. Hence, the sample still has the potential to be strengthened further during a second sintering (healing). If the re-sintering is applied after the sample has already experienced some damage under mechanical loading (like uni-axial tension or compression), the question is to which extent the damage can be healed.

In Section 2 the technical details of the simulation method are summarized, and the pressure-sintering contact model is described. In the following section 3, numerical results of DEM simulations are reviewed for damage under unconfined uni-axial tensile and compressive loading. In Section 3.3 a qualitative comparison is made between the numerical data and results from laboratory uni-axial compression tests on unconfined asphalt samples [15]. Finally in Section 3.4, DEM simulations are reported, where self-healing through re-sintering is applied to the previously used numerical samples. 


\section{Simulation method}

\subsection{DEM modelling of particulate materials}

The Discrete Element Method (DEM) [3] for particle systems can be used to illustrate how the macroscopic response of a solid-like, sintered sample, resembling an asphalt mixture, depends on various micro- and meso-scopic properties such as the particle-particle contact network, the particle size, and the contact adhesion between particles (simulating the interaction of the bitumen with the particles), their contact friction and stiffness. In the present work, after solving the equations of motion at the particle level, the coupling between micro- and macro scale properties is performed. The response of the particle system (expressed in terms of macroscopic stress and strain) is obtained by averaging [18] the local quantities at the particle contact level (interparticle contact forces and displacements) over the assembly. The effective response then depends directly on the chosen particle contact model [11][19][20] Even though recent studies have demonstrated that the accurate simulation of systems composed of non-spherical particles is possible [21][22], for simplicity we restrict ourselves here to spherical particles.

\subsection{Sintering contact model}

In the following, particulate material samples are (i) prepared, (ii) deformed and damaged, and then (iii) self-healed and (iv) deformed again. The non-linear model by Luding et al. [11][23][24] is used - see these references for more details, to describe the particle-particle interaction in the mixture. In Figure 1, the normal contact force $f$ (that is directed parallel to the line connecting the centers of two contacting particles) is plotted against the contact overlap (resembling the deformation between particles at the contact), $\delta>0$. If $\delta<0$, there is no contact between particles, and thus $f=0$. This sign convention relates positive (negative) values of the contact displacement $\delta$ to overlap/deformation (separation), while positive (negative) values of the contact force $f$ relate to compression/repulsion (tension/attraction).

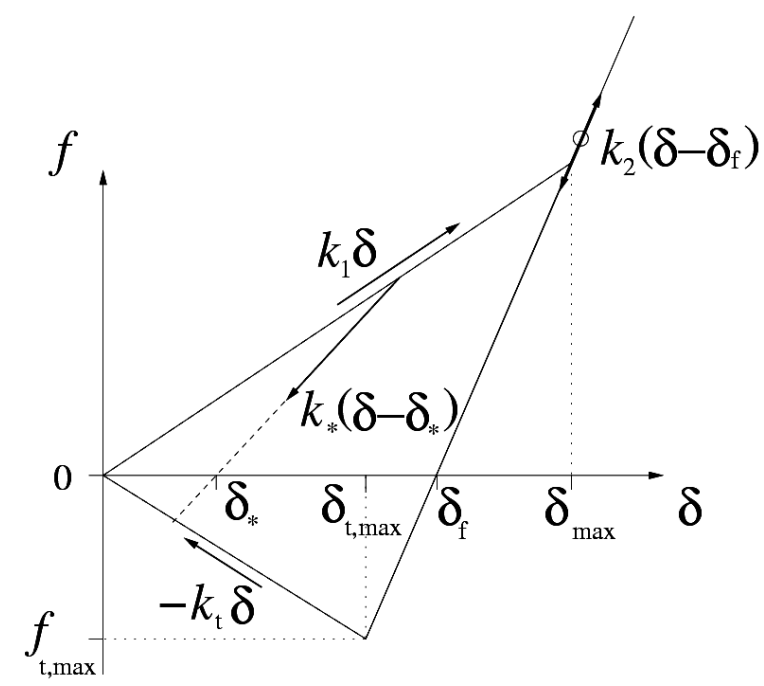

Fig. 1: Particle contact model plotted as force-displacement relation [11][23], with $\delta$ for contact positive, and repulsion and attraction forces positive and negative, respectively.

A contact begins at $\delta=0$ and, during initial compressive loading, the contact force increases with the overlap as $f=k_{1} \delta$, with $k_{1}$ the elasto-plastic contact stiffness. When the external compressive forces are compensated by the contact repulsive force at the maximum contact overlap, $\delta_{\max }$, for unloading, the contact stiffness increases to a value $k_{2}$, so that the elastic unloading force is $f=k_{2}\left(\delta-\delta_{\mathrm{f}}\right)$. Elastic unloading to zero contact force leads to the (plastic) contact overlap $\delta_{\mathrm{f}}$ 
$=\left(k_{2}-k_{1}\right) \delta_{\max } / k_{2}$. If the overlap is further decreased, the contact force gets tensile, with maximum tensile contact force $f_{\mathrm{t}, \max }=-k_{\mathrm{t}} \delta_{\mathrm{t}, \max }$, realized at contact displacement $\delta_{\mathrm{t}, \max }$.

For the sake of brevity, the tensile softening parameter $k_{\mathrm{t}}$ hereafter is referred to as the "contact adhesion". Note that, mostly for practical reasons [11], for contact deformations above $\delta_{\max }$, the force follows the limit branch $f=k_{2}\left(\delta-\delta_{\mathrm{f}}\right)$, since further loading is unrealistic anyway and would lead to much stiffer behavior if properly modeled. The extreme loading and unloading limit branches are reflected by the outer triangle in figure 1. Starting from the realized maximal overlap, $\delta_{\max }{ }^{*}<\delta_{\max }$, unloading occurs within the outer triangle, as characterized by a branch with stiffness $k_{*}=k_{1}+\left(k_{2}-k_{1}\right) \delta_{\max }{ }^{*} / \delta_{\max }$, and (elastic, reversible) force, $f^{*}=k_{*}\left(\delta-\delta^{*}\right)$. The intermediate stiffness $k_{*}$ follows from a linear interpolation between $k_{1}$ and $k_{2}$ - which is our (arbitrary) choice due to the lack of experimental data on this (probably) non-linear behavior. In summary, the model has three ("stiffness") $k$-parameters that describe the three relevant physical effects at the contact: (1) elasticity, (2) plastic deformations, and (3) contact-adhesion. Furthermore, the model involves (4) a non-linear contact stiffness via the choice of $k_{*}$. This piece-wise linear model is a compromise between simplicity and the need to model physical effects. Except for some early theoretical studies, see [20] and the many works that are based on it, there is no experimental/numerical literature available to our knowledge that provides enough detailed information on the force-displacement relations, involving all four physical contact properties above and their nonlinear, history-dependent behavior. If this information becomes available, the present model can be extended and generalized.

The tangential contact force acts parallel to the particle contact plane and is related to the tangential contact displacement through a linear elastic contact law, with the tangential stiffness $k_{\mathrm{s}}$. The tangential contact displacement depends on both the translations and rotations of the contacting particles. Coulomb friction determines the maximum value of the tangential contact force: During sliding the ratio between the tangential contact force and the normal contact force is assumed to be limited and equal to a (constant) dynamic friction coefficient $\mu_{\mathrm{d}}$; during sticking, the tangential force is limited by the product of normal force and static coefficient of friction $\mu_{\mathrm{s}}$.

\begin{tabular}{|l|l|l|l|}
\hline \multicolumn{1}{|c|}{ Property } & \multicolumn{1}{|c|}{ Symbol } & \multicolumn{1}{c|}{ Values [14] } & \multicolumn{1}{c|}{ SI units } \\
\hline Time Unit & $t_{u}$ & 1 & $1 \mathrm{~ms}$ \\
\hline Length Unit & $x_{u}$ & 1 & $1 \mathrm{~m}$ \\
\hline Mass Unit & $m_{u}$ & 1 & $1 \mathrm{~kg}$ \\
\hline Average radius & $\bar{R}$ & $0.510^{-3}$ & $0.5 \mathrm{~mm}$ \\
\hline Material density & $\rho$ & 2000 & $2000 \mathrm{~kg} / \mathrm{m}^{3}$ \\
\hline Elastic stiffness & $k=k_{2}$ & $5.10^{4}$ & $5.10^{10} \mathrm{~kg} / \mathrm{s}^{2}$ \\
\hline Plastic stiffness & $k_{1} / k_{2}$ & 0.5 & \\
\hline Adhesion "stiffness" & $k_{t} / k_{2}$ & {$[0, \ldots, 20]$} & \\
\hline Friction stiffness & $k_{s} / k_{2}$ & 0.2 & \\
\hline Plasticity range & $\phi_{f}$ & 0.05 & \\
\hline Coulomb friction & $\mu=\mu_{d}=\mu_{s}$ & 1 & $5.10^{6} \mathrm{~kg} / \mathrm{s}$ \\
\hline Normal viscosity & $\gamma=\gamma_{n}$ & $5.10^{3}$ & \\
\hline Tangential viscosity & $\gamma_{f} / \gamma$ & 0.2 & \\
\hline Background viscosity & $\gamma_{b} / \gamma$ & 4 & \\
\hline Background torque & $\gamma_{b r} / \gamma$ & 1 & \\
\hline
\end{tabular}

Tab. I: Microscopic material parameters based on Ref. [11], but rescaled as indicated in the right column.

\section{Results}

The simulations reported here consist of four subsequent stages, namely (i) a sample preparation stage (isotropic compression), (ii) a uni-axial (tensile or compressive) loading stage, (iii) a self-healing stage, and (iv) the continuation of the uni-axial loading.

Six plane, perpendicular outer walls form a cuboidal volume, with side lengths of $L=11.5 \mathrm{~mm}$. The samples are composed of about $10^{3}$ poly-disperse spherical particles (see Fig 2 as an example), with particle radii drawn from a Gaussian distribution around mean $\bar{R}=0.5 \mathrm{~mm}[11][25]$. The particle density used in the simulations is $\rho=2000 \mathrm{~kg} / \mathrm{m}^{3}$, 
the maximum elastic contact stiffness is $k_{2}=5 \cdot 10^{10} \mathrm{~N} / \mathrm{m}$. The initial elasto-plastic stiffness (normalized by $k_{2}$ ) is $k_{1} / k_{2}=1 / 2$, and the contact adhesion $k_{t} / k_{2}$ is varied. The other stiffness parameters, friction coefficients, and viscous damping parameters are summarized in Table I, most of them being dimensionless, like all quantities discussed in the rest of the paper. As final remark, we note that the choice of parameters is empirical - most of them kept fixed here, only adhesion is varied systematically.
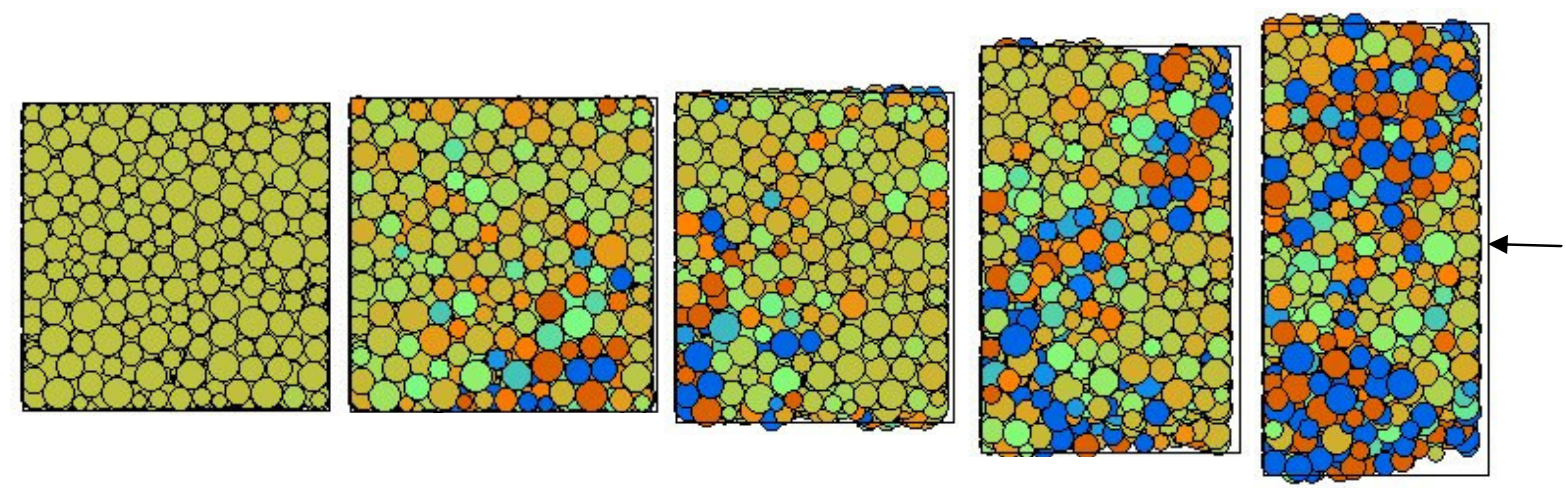

Fig. 2: Sequence of snapshots from a compression test with poly-disperse particles and rigid outer walls $\left(\mathrm{k}_{\mathrm{t}} / \mathrm{k}_{2}=0.5\right)$. The circles are the particles with the greyscale coding the average stress.

\subsection{Isotropic Loading}

In this section the sample preparation by pressure sintering [23] is reported. During sintering, the particles deform plastically at contact and stick to each other due to strong, non-linearly increased van der Waals forces. At the same time, the sample shrinks, i.e. becomes denser. Such pressure-sintering results in a solid sample with bonded particles, similar to asphalt mixtures. Moreover, the sintering model can be temperature-dependent, resembling the effect of the temperature in the asphalt preparation with bitumen [12]. The process is characterized by two stages: the first stage reflects the application of a hydrostatic (or isotropic) pressure, $p_{\mathrm{s}} / \sigma_{0}=4.10^{-2}$, to a loose assembly of particles, with the reference stress $\sigma_{0}=k_{1} /(2 \bar{R})$. This desired isotropic stress is slowly applied to the six outer walls. The hydrostatic loading process is considered to be finished when the kinetic energy of the sample is negligible compared to the potential energy. For our sample, the solid volume fraction (volume of the solid particles over total volume) at the end of the hydrostatic loading process is $v=0.676$ (which relates to a porosity (volume of fluid and air phases with respect to total volume, in the specific case of asphalt) of $1-v=0.324)$.

The second stage of the pressure sintering process is reflected by a stress relaxation phase, where the external hydrostatic pressure is strongly reduced, while the adhesion between particles is now made different then zero. Due to the presence of particle contact adhesion, the lateral stability of the specimen remains preserved when the hydrostatic pressure is released, i.e., a coherent and stable particulate structure is obtained that can be subsequently used in the analysis of damage and healing under uni-axial loading conditions. The solid volume fraction of the sample after stress relaxation is decreased to $v=0.63$.

\subsection{Response under uni-axial compression and tension}

In the uni-axial compression (tension) test, one of the two outer walls, with its normal parallel to the axial (loading) direction, is slowly moved towards (away from) the opposite wall. The change of the wall displacement in time is prescribed by a cosine function with rather large period in order to limit inertia effects.

The response of the sample under uni-axial compression and uni-axial tension is shown in Figure 2. The normal axial stress $\sigma$, normalized by the reference stress $\sigma_{0}$, is plotted as a function of the normal axial strain $\varepsilon$, where positive stress (strain) values relate to compression (contraction). The stress-strain curves are depicted for different values of $k_{\mathrm{t}}$ (normalized by $k_{2}$ ), which quantifies the adhesion at the particle contacts, see Figure 1. A larger particle contact adhesion increases the effective strength of the sample, both under uni-axial tension and compression. Furthermore, the overall strain at which the effective stress reaches its maximum increases with increasing contact adhesion, $k_{\mathrm{t}}$.

Note that the maximum stress under uni-axial compression is order of five times larger than under uni-axial tension. A relatively high compressive strength in relation to the tensile strength is typical of various sintered materials, such as ceramics [26], and appears even in asphalt mixtures [15][27]. As further result, from Figure 2, the softening branch under uni-axial tension is somewhat steeper than under uni-axial compression. Here we use a rate that is close to the 
quasi-static regime, as studied in more detail in [28]. The initial loading branch is linear up to large stress and the initial (elastic) axial stiffnesses $C_{\mathrm{t}}$ in tension and compression are determined by the sample preparation procedure, and are approximately equal for all the cases considered here, i.e., $C_{t} / \sigma_{0}=1.04$. The tensile responses are all characterized by local failure at the center of the sample.

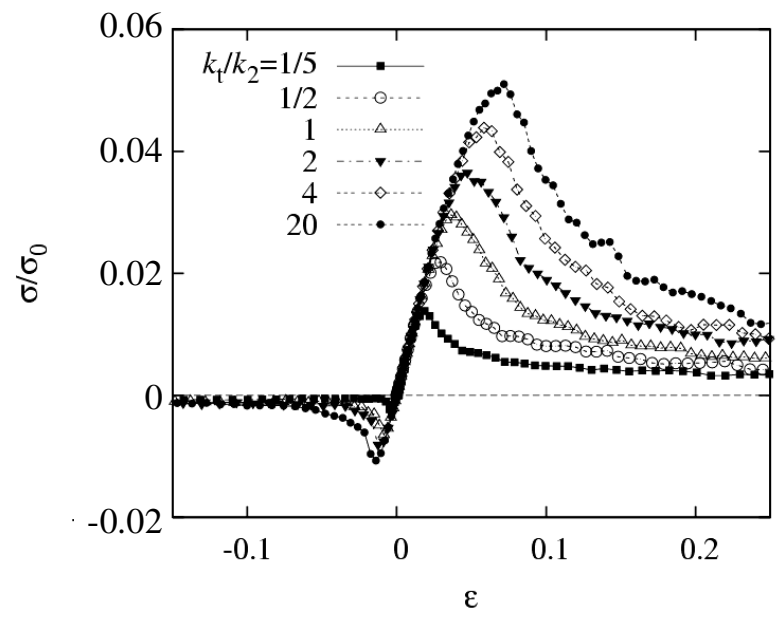

Fig. 3: Dimensionless axial stress (normalized by $\sigma_{0}=k_{1} /(2 \bar{R})$ ), plotted against axial strain during uni-axial compression (positive stress and strain values) and uni-axial tension (negative stress and strain values), for different particle contact adhesions $k_{\mathrm{t}} / k_{2}$ (after [14]).

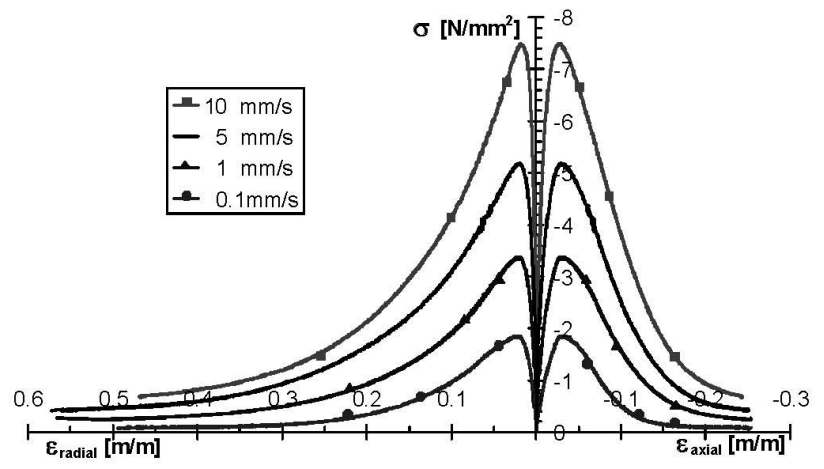

Fig. 4: Axial stress $\sigma$ versus axial strain $\varepsilon_{\text {axial }}$ and radial strain $\varepsilon_{\text {radial }}$ for uni-axial compression tests on DAC samples at different strain rates and $\mathrm{T}=30^{\circ} \mathrm{C}$ (after [15]). Negative $\sigma$ means here compression, while negative and positive $\varepsilon$ mean axial and radial strain, respectively.

\subsection{Comparison between numerical simulations and physical experiments}

We refer to experiments in [15] to qualitatively compare our numerical investigation with laboratory tests on asphalt mixtures. The authors carry on uni-axial unconfined compression tests on compacted samples of dense asphalt concrete (DAC 0/5). The tests are performed in the displacement control mode, at constant axial deformation rates (refer to [15] for details). Mixtures such as DAC are continuously graded (in agreement with our numerical sample) and derive their stability from the packing of the aggregates and the cohesion provided by the bitumen. We report in Fig. 3 the stressstrain behavior for the aggregate compressed at different strain rate. Despite the differences between the numerical and laboratory tests, the comparison of Figs. 2 and 3 (axial stress versus axial strain on the right side of Fig.3) shows that 
the elasto-plastic adhesive contact model is able to capture qualitatively the basic features in the behavior of the asphalt mixture, but the strain-rate dependence has to be studied further.

\subsection{Self-healing under uni-axial compression and tension}

In this section we show a possible modeling of induced self healing [13] mechanisms in asphalt. During uni-axial compression, the sample is stopped at various strains, see Figure 4, and the self-healing is achieved by an instantaneous increase of the particle contact adhesion $k_{\mathrm{t}}$, which is assumed to be the net-effect of a re-sintering cycle, like warming up an asphalt mixture. Technically, on the contact level, an increase of the contact adhesion $k_{\mathrm{t}}$ corresponds to an increase of the maximum tensile strength $f_{\mathrm{t}, \max }$, and a decrease of the corresponding displacement $\delta_{\mathrm{t}, \text { max }}$, i.e. rupture occurs at large tensile strain.

After the application of the re-sintering cycle (self-healing), uni-axial loading is resumed, where the effect of selfhealing on the effective stress-strain response of the sample becomes apparent through a comparison of its response with that of both, the unhealed reference sample and a pre-emptively healed sample, which has the stronger contact adhesion from the beginning on.

Under uni-axial compression, see Fig. 4(a), or uni-axial tension, see Fig. 4(b), the self-healing of the initial sample with $k_{\mathrm{t}} / k_{2}=1 / 5$ is activated by instantaneously increasing the contact adhesion to $k_{\mathrm{t}} / k_{2}=1$ or 20 , uniformly at all particle contacts. Figure 4 shows the response curves after the initiation of self-healing (dashed lines, labeled with the abbreviation ' $\mathrm{SH}$ '), together with the stress-strain responses of the relatively weak $\left(k_{\mathrm{t}} / k_{2}=1 / 5\right.$, solid squares), strong $\left(k_{\mathrm{t}} / k_{2}=1\right.$, triangles) and very strong $\left(k_{\mathrm{t}} / k_{2}=20\right.$, solid circles) samples. The maximum compressive strength reached after self-healing is larger for healing at smaller deformation - and thus smaller damage. Astonishingly, for all selfhealing cases considered, the response eventually converges to the response of the "strong" sample with $k_{\mathrm{t}} / k_{2}=1$ or 20 . The strong sample stress-response thus can be interpreted as the response of a pre-emptively "self-healed" sample, where the increase in contact adhesion is initiated at the onset of mechanical loading already. The response of the sample with $k_{\mathrm{t}} / k_{2}=1$ or 20 acts as envelope for the responses of the self-healed samples with $k_{\mathrm{t}} / k_{2}$ increased from $1 / 5$ to 1 or 20 , respectively.
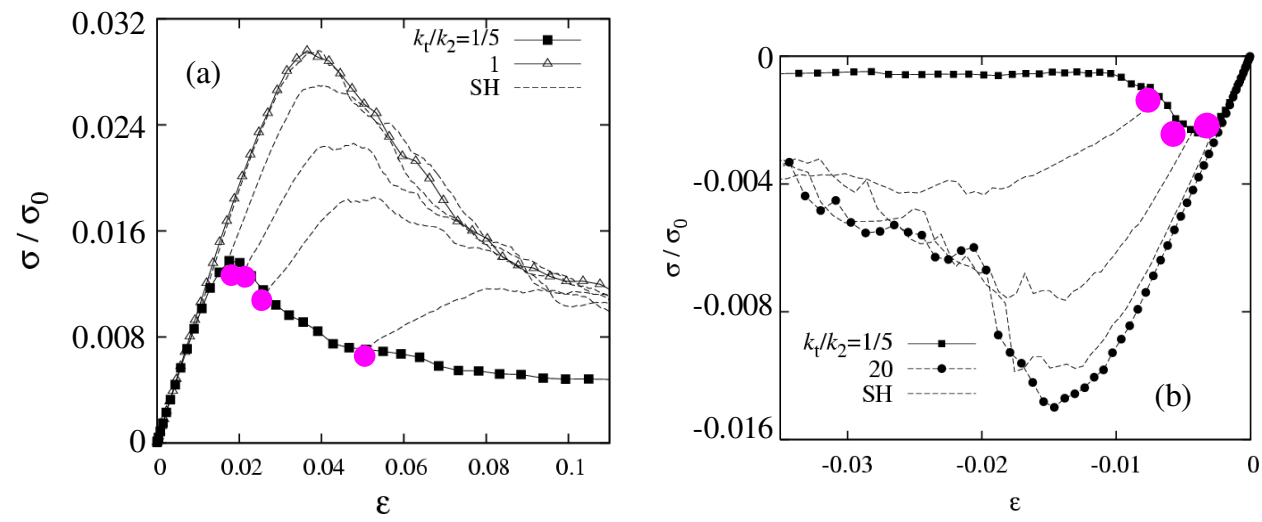

Fig. 5: Axial stress versus axial strain during (a) uni-axial compression and (b) uni-axial tension, for particle contact adhesions $k_{\mathrm{t}} / k_{2}=1 / 5$ (solid squares). The initiation of self-healing occurs at axial strains $\varepsilon \approx 0.019,0.022,0.026$, and 0.045 for compression and $\varepsilon \approx-0.003,-0.005$, and -0.007 for tension, as indicated by (magenta) dots. The self healing strength is activated (a) five times and (b) hundred times larger than the original samples' contact adhesion. The selfhealing stress responses are given (starting from the different strain points) by the dashed lines. The outer envelope corresponds to self-healing (increase of contact adhesion) already at zero strain (after [14]). 


\section{Conclusions}

In this work, (1) isotropic preparation, (2) uni-axial deformation, and (3) self-healing processes in damaged adhesive granular assemblies have been studied using DEM simulations with the final goal of application to asphalt mixtures. The effect of self-healing is mimicked by a (global) sintering process, as modeled by increasing the particle contact adhesion from relatively "weak" to rather "strong".

After isotropic compression, uni-axial compression/tension was applied to the sample for different contact adhesion strengths. While the initial stiffness (slope of the stress-strain curves) is not much affected, the peak strength of the material is: The stronger the contact adhesion, the larger the peak strength. For compression, the strength appears about a factor of five larger than for tension and the softening branch is rather smooth for compression, while the tensile regime shows a sharper drop, resembling more brittle-like behavior.

Moreover, at different strains, the uni-axial deformation is stopped and global self-healing is applied. The stress-strain response obtained from such self-healed samples eventually converges to the envelope curve that represents the damage response of a sample that has the "strong" contact adhesion since the onset of loading. Another result is that the maximum sample strength reached after self-healing very much depends on the deformation level at which self-healing is activated.

This preliminary work shows how powerful DEM simulations can be in describing the constitutive behavior of asphalt mixtures. In fact, discrete simulations give insights on the material microstructure and link observable (macroscopic) phenomena with the kinematics of the interacting components. Very useful information can be extracted from such microscopic analysis, e.g. on how and when act to induce self-healing in a damaged material.

Future work concerns an interesting investigation issue: the easy switching between adhesive and non-adhesive contact forces in our numerical simulations allows us to separately investigate the role of the single components in the asphalt mixture, focusing in particular on the aggregate skeleton. Through our tool, we want to study how differences in dimension and arrangement of the aggregate skeleton affect the behavior of the full mixture [27][29].

\section{References}

[1] Simulation of HMA compaction by using FEM, ter Huerne H. L., van Maarseveen M. F. A. M., Molenaar A. A. A., and van de Ven M. F. C., Int. J. Pavement Engineering 9:3,153-163, 2008

[2] Review of advances in micromechanical modeling of aggregate-aggregate interactions in asphalt mixtures, You Z., Dai Q., Canadian Journal of Civil Engineering, 34, 239-252, 2007.

[3] A discrete numerical model for granular assemblies, Cundall P.A. and Strack O.D.L., Geotechnique, 29,47-65, 1979.

[4] Micromechanical modeling for behavior of cementitious granular materials, Zhong X and Chang C.S., ASCE Journal of Engineering Mechanics, 125:11, 1280-1285, 1999.

[5] Micromechanical modeling of asphalt concrete in connection with pavement rutting problems, Rothenburg, L., Bogobowicz, A., Haas, R., Proceedings of the 7th International Conference on Asphalt Pavements I, 230-245, 1992.

[6] Simulation of the behavior of asphalt concrete using discrete element method, Chang, G.K., Meegoda, N.J., In: Proc.2nd Int. Conf. Discr. Element Meth., MIT, 437-448, 1993.

[7] A comparison of micro-mechanical modeling of asphalt materials using finite elements and doublet mechanics, Sadd, M.H., Dai, Q. Mechanics of Materials 37, 641-662, 2005.

[8] Discrete element modeling of asphalt concrete: micro-fabric approach, Buttlar, W.G., You, Z., TRR, No. 1757, 111-118, 2001.

[9] A study of failure in cohesive particulate media using the discrete element method, Ullidtz, P., in: Proc. 80th TRB Meeting, Washington, DC, 2001.

[10] Finite-element analysis and experimental study on tensile fatigue behaviour of bitumen-stone adhesion. Fatigue \& Fracture of Engineering Materials and Structures, Mo L. T., Huurman M., Wu, S. P. Molenaar A. A. A., 30:9, 823831, 2007.

[11] Cohesive, frictional powders: contact models for tension, Luding, S., Granular Matter 10, pp. 235-246, 2008. 
[12] A discrete model for long time sintering, Luding, S., Manetsberger, K., Muellers, J., Journal of the Mechanics and Physics of Solids 53:2, 455-491, 2005.

[13] An introduction to material design principles: Damage prevention versus damage management. in: Self Healing Materials. An Alternative Approach to 20 centuries of Materials Science, Van der Zwaag S., Van der Zwaag ed., Springer, Dordrecht, NL, 1-18, 2007.

[14] From discrete particles to solids - about sintering and self-healing (Review), Luding S., Comp. Methods in Mater. Science 11:1, 53-63, 2011.

[15] Asphalt Concrete Response (ACRe) - determination, modeling and prediction, Erkens S.M.J.G, Ph.D. thesis Delft University of Technology, 2002.

[16] Improvement of mechanical strength of $8 \mathrm{~mol} \%$ Yttria-stabilized zirconia ceramics by spark-plasma sintering, Takeuchi, T., Kondoh, I., Tamari, N., Balakrishnan, N., Nomura, K., Kageyama, H., Takeda, Y., Journal of The Electrochemical Society 149, A455-A461, 2002.

[17] In situ strength evolution during the sintering of bronze powders, Shoales, G. A., German, R. M., Metallurgical and Materials Transactions A 29a, 1257-1263, 1998.

[18] Micro-macro transition for anisotropic, frictional granular packings, Luding, S., International Journal of Solids and Structures 41, 5821-5836, 2004.

[19] Quasi-static deformation of a soft particle system, Thornton, C., Antony, S. J., Powder Technology 109, 179-191, 2000 .

[20] Assessment of mechanical properties of cohesive particulate solids - part 1: particle contact constitutive model, Tomas, J., Particle Science Technology 19, 95-110, 2001.

[21] Role of anisotropy in the elastoplastic response of a polygonal packing, Alonso-Marroquin, F., Luding, S., Herrmann, H. J., and Vardoulakis, I., Phys. Rev. E 71, 051304, 2005.

[22] On the application of a discrete model to the fracture process of cohesive granular materials, D'Addetta, G. A., Kun, F., Ramm, E., Granular Matter 4:2, 77-90, 2002.

[23] Self-healing of damaged particulate materials through sintering, Luding, S., Suiker, A., Philosophical Magazine 88: 28-29, 3445-3457, 2008.

[24] Evolution of swelling pressure of cohesive-frictional, rough and elasto-plastic granulates, in: Geomechanics and Geotechnics: From Micro to Macro, Luding, S., and Bauer, E., In: IS-Shanghai Conference Proceedings, 11.-12. Oct. 2010, M. Jiang, L. Fang, and M. Bolton (Eds.), CRC Press/Balkema, NL, 495-499, 2010.

[25] Modelling particulate self-healing materials and application to uni-axial compression, Herbst, O., Luding, S., Int. J. of Fracture 154, 87-103, 2008.

[26] Ceramic Microstructures, Property Control by Processing, Lee, W. E., Rainforth, W. M., Chapman and Hall, London, UK, 1995.

[27] Permanent deformation of asphalt mixes, Muraya P.M., Ph.D. thesis - Delft University of Technology, 2007.

[28] Modelling particulate self-healing materials and application to uni-axial compression, Herbst, O., Luding, S., Int. J. of Fracture 154, 87-103, 2008.

[29] On the equation of state near the jamming transition for polydisperse, smooth, elastic spheres, Ogarko V. and Luding S., submitted, 2011. 